Journal of Applied Pharmaceutical Science Vol. 7 (04), pp. 053-060, April, 2017

Available online at http://www.japsonline.com

DOI: $10.7324 / \mathrm{JAPS} .2017 .70407$

ISSN 2231-3354 (cc) BY-NC-SA

\title{
Identification of new compounds from Fumaria parviflora Lam.
}

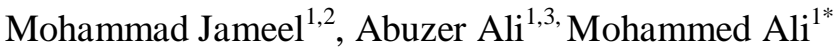 \\ ${ }^{1}$ Phytochemistry Research Laboratory, Department of Pharmacognosy and Phytochemistry, Faculty of Pharmacy, Jamia Hamdard, New Delhi, India. \\ ${ }^{2}$ Regional Research Institute of Unani Medicine, Central Council of Research Unani Medicine, Aligarh-202001, India. \\ ${ }^{3}$ Department of Natural Products and Alternative Medicine, College of Clinical Pharmacy, University of Dammam, Dammam, Kingdom of Saudi Arabia.
}

\begin{tabular}{|c|c|}
\hline ARTICLE INFO & ABSTRACT \\
\hline $\begin{array}{l}\text { Article history: } \\
\text { Received on: } 21 / 02 / 2017 \\
\text { Accepted on: } 27 / 03 / 2017 \\
\text { Available online: } 30 / 04 / 2017\end{array}$ & $\begin{array}{l}\text { Fumaria parviflora Lam. (Fumariaceae) is a traditional medicinal herb chiefly used as a blood, skin and liver } \\
\text { related disorder along with several human ailments like abdominal cramps, diarrhea, fever, antidyspeptic, } \\
\text { cholagogue, diaphoretic, diuretic, laxative, sedative, tonic and syphilis. Phytochemical investigation of a } \\
\text { methanolic extract of the plant of the plant led isolation of six new compounds characterized as 2- }\end{array}$ \\
\hline $\begin{array}{l}\text { Key words: } \\
\text { Isolation, Extraction, } \\
\text { Traditional medicine, } \\
\text { Homaira, Shahtara, Herbal. }\end{array}$ & 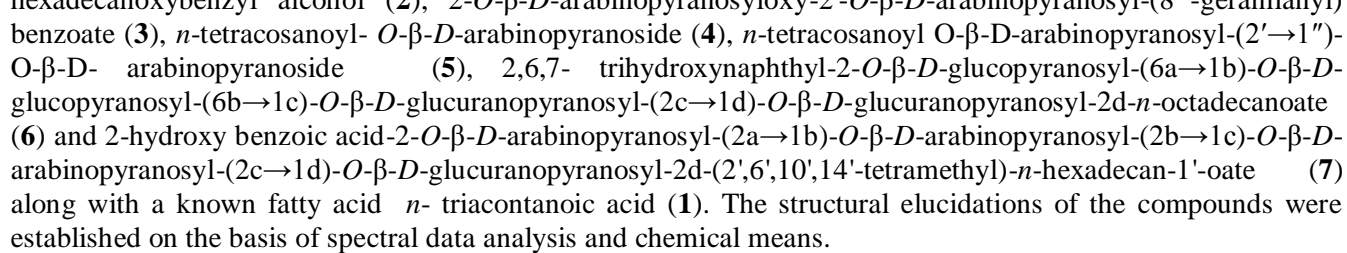 \\
\hline
\end{tabular}

\section{INTRODUCTION}

Fumaria parviflora Lam., (Fumariaceae) is widely used herb in folkloric as well as traditional system of medicine with several synonyms like earth smoke, beggary, wax dolls in English (Orhan et al., 2010), Pitpapra in Hindi, Shahtrah in Urdu (Chopra et al., 2002) and Homaira in Arabic (Mossa et al., 1987). It is small, annually and wildly growing weedy herb in agricultural fields, plains and lower hills (Suau et al., 2002). It is $10-40 \mathrm{~cm}$ in height (Kirtikar and Basu, 1999) and consists of 18 genera and 450 species globally and mainly distributed in North America, Europe, Asia and Africa (Mabberley, 2008). It is imported from Persia as a chief ingredients of blood purifying

* Corresponding Author

Mohammed Ali, Phytochemistry Research Laboratory, Department of

Pharmacognosy and Phytochemistry, Faculty of Pharmacy, Jamia

Hamdard, New Delhi- 110062, India. Email: maliphyto @ gmail.com agent and used for skin diseases in Unani system of medicine (Khare, 2004). It is considered useful to treat abdominal cramps (Duke et al., 2002), diarrhea, fever (Haq and Hussain, 1993), cholagogue, diaphoretic, diuretic, laxative, sedative, tonic, jaundice and leprosy (Nadkarni, 1976), antidyspeptic (Anonymous, 2006) and tuberculosis (Anonymous, 2005) due to presence of several vital secondary metabolites including flavonoids, tannins, saponins, phenolic compounds (Naz et al., 2013), alkaloids (Popova et al., 1982; Rahman et al., 1992; Suau et al., 2002), glycosides (Hussain et al., 1980) citric, coumaric, ferulic, fumaric, malic, 3-hydroxybenzoic, protocatechuic and caffeic acids (Sousek et al., 1999). Traditional medicines have undeniably result oriented, diversifying, inventive and successful approach for discovering novel crucial unmatched lead molecules that might be transform in practice to accept the future challenges (Fakim, 2006). Therefore an integrated approach towards the isolation of new chemical entities with ethnomedicinal implications for promotion and development of traditional medicine by column chromatographic methods has been made. 
Previously isolation of n-octacosan-7 $\beta$-ol (Jameel et al., 2014A), n-propyl-3,4-dioxymethylene benzene 5 $\beta, 6,7,8,9,10 \beta$ hexahydrocoumarin and 2,6-dimethyl dodecan-10-oyl-12,15-olide (Jameel et al., 2014B), (5 $\alpha \mathrm{H}, 11 \alpha \mathrm{H})-8$-oxo-homoiridolide, $n$ docosanyl-2- $O-\beta-D$-glucopyranosyl salicylate, 2-methyl-6hydroxymethylenedodecan-10-oyl-12, 15-olide14- $O-\beta-D$ xylopyranoside, 4-oxo-stigmast-5-en-3 $\beta$-ol- $D$-glucopyranoside and salicylic acid- $O-\beta-D$-xylopyranoside (Jameel et al., 2014C) have been done from this plant grown in Delhi region.

\section{MATERIAL AND METHODS}

General

UV spectra were measured with a Lambda Bio 20 Spectrophotometer (Perkin Elmer, Rotkreuz, Switzerland) in methanol. The ${ }^{1} \mathrm{H}(400 \mathrm{MHz})$ and ${ }^{13} \mathrm{C}(100 \mathrm{MHz}) \mathrm{NMR}$ spectra were recorded on a Bruker ARX-Spectrometer (Rheinstetten, Baden-Wurttemberg, Germany), using $\mathrm{CDCl}_{3}$ and DMSO- $\mathrm{d}_{6}$ as solvents and TMS (Fluka analytical, Sigma-Aldrich, Netherland) as an internal standard. Melting points were determined by a thermoelectrically heated Perfit melting point apparatus (Ambala, India) without correction. Infra Red (IR) spectra were recorded using $\mathrm{KBr}$ pellets with a Jasco FT-IR-5000 Spectrometer (FTS 135, Kawloon, Hong Kong). Mass-spectrometric detection was carried out on ESI MS (Q-TOF-ESI) (Waters Corp., Manchester, UK), an electrospray-ionisation (ESI) technique with positive ionization mode. Column chromatography was performed on silica gel (Qualigens, Mumbai, India), 60-120 mesh and solvents taken were purchased from Merck Specialties (E. Merck, Pvt. Ltd. New Delhi, India). Pre-coated Aluminum TLC plates of silica gel 60 $\mathrm{F}_{254}$ (Merck, Darmstadt, Germany) were used to run and spots were visualized by exposure to iodine vapors, and UV radiations and spraying with anisaldehyde- sulphuric acid solution.

\section{Methods \\ Plant material}

The $F$. parviflora whole plant was collected from the herbal garden of Jamia Hamdard, New Delhi and identified by Prof. Javed Ahmad, In-charge of the herbal garden. A specimen voucher of the drug was deposited in the herbarium of the Faculty of Pharmacy with a reference number PRL-JH/2011/05.

\section{Preparation of extract and isolation}

The dried $F$. parviflora whole plant $(2.5 \mathrm{~kg})$ was coarsely powdered and extracted with methanol for $48 \mathrm{~h}$ using a Soxhlet extractor in hot and cold cycle of interval of 5-6 hrs. The extract was dried under reduced pressure to obtain a dark brown residue $(380 \mathrm{~g})$. The extract was partioned with hexane (750 $\mathrm{ml} \mathrm{X} \mathrm{3)}$ ) and chloroform (500 $\mathrm{ml} \mathrm{X} \mathrm{3)} \mathrm{and} \mathrm{excluded.} \mathrm{The} \mathrm{remaining} \mathrm{portion} \mathrm{of}$ extract $(80 \mathrm{~g})$ was dissolved in minimum amount of methanol and adsorbed mechanically by heating on water bath with column grade silica gel (60-120 mesh) to obtain a slurry that was chromatographed over silica gel column loaded in chloroform and the eluants of each fraction were examined by precoated
Aluminum TLC plate. The column was eluted with gradient mixtures of chloroform-methanol (99:1, 97:3, 19:1, 93:7, 9:1, 3:1, $v / v)$ to isolate the compounds 1-7.

Further a spot of TLC chromatogram of methanolic extract was scraped out, sonicated to $2-3 \mathrm{~min}$ at $30{ }^{\circ} \mathrm{C}$ in MS grade methanol and filtered first whatman's filter paper and then 0.2 sized $\mu$ filter for Mass spectrometer. Its molecular weight and $\mathrm{m} / \mathrm{z}$ fragments were analyzed and compared with the same $R_{f}$ of eluants of column which is showed in Fig.1.

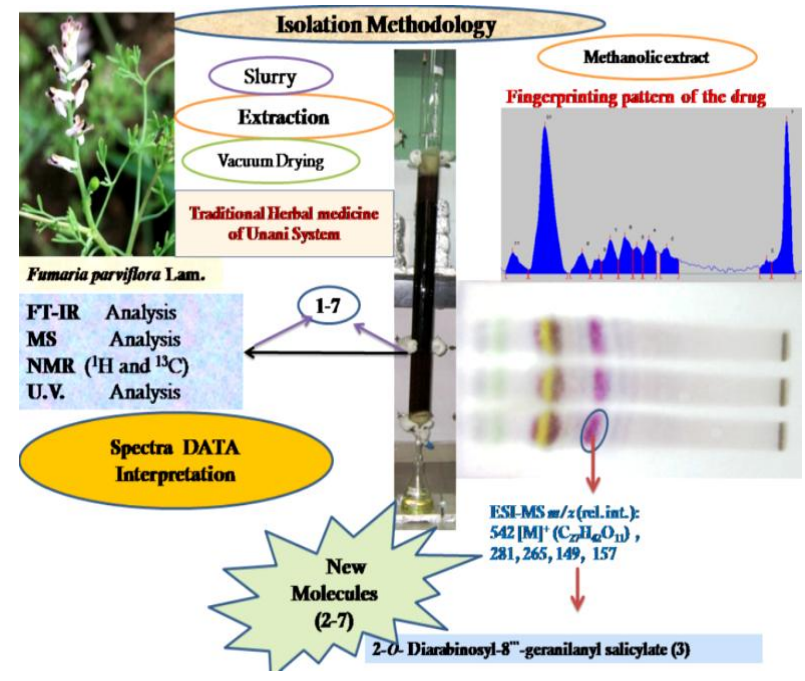

Fig. 1: Layout of Isolation.

\section{RESULTS}

\section{Melissic acid (1)}

Elution of the column with chloroform-methanol (99:1, $v / v)$ gave colorless amorphous mass of $\mathbf{1}$, purified from chloroform-methanol $(1: 1, v / v), 652 \mathrm{mg}(0.052 \%$ yield), m.p. 92$93{ }^{\circ} \mathrm{C} ; \mathrm{R}_{\mathrm{f}} 0.7$ (chloroform), UV $\lambda_{\max }(\mathrm{MeOH}): 206,223 \mathrm{~nm}(\log \varepsilon$ 3.7, 1.8). IR $v_{\max }(\mathrm{KBr}): 3485,2924,2854,1704,1458,1255$, $1178,974,725 \mathrm{~cm}^{-1} ;{ }^{1} \mathrm{H}$ NMR $\left(\mathrm{CDCl}_{3}\right): \delta 2.28(2 \mathrm{H}, \mathrm{t}, J=7.2 \mathrm{~Hz}$, $\left.\mathrm{H}_{2}-2\right), 1.99\left(2 \mathrm{H} \mathrm{m}, \mathrm{CH}_{2}\right), 1.57$ (4H, brs, $\left.2 \times \mathrm{CH}_{2}\right), 1.21$ (48 H, brs, $\left.24 \times \mathrm{CH}_{2}\right), 0.83(3 \mathrm{H}, \mathrm{t}, J=6.8 \mathrm{~Hz}, \mathrm{Me}-1) .{ }^{13} \mathrm{C} \mathrm{NMR}\left(\mathrm{CDCl}_{3}\right): \delta$ 179.18 (C-1), $39.30(\mathrm{C}-2), 31.74\left(\mathrm{CH}_{2}\right), 29.68\left(10 \times \mathrm{CH}_{2}\right), 29.64$ $\left(5 \times \mathrm{CH}_{2}\right), 29.59\left(\mathrm{CH}_{2}\right), 29.43\left(\mathrm{CH}_{2}\right), 29.34\left(\mathrm{CH}_{2}\right), 29.24\left(\mathrm{CH}_{2}\right)$, $29.07\left(\mathrm{CH}_{2}\right), 27.96\left(\mathrm{CH}_{2}\right), 27.71\left(\mathrm{CH}_{2}\right), 27.42\left(\mathrm{CH}_{2}\right), 27.19\left(\mathrm{CH}_{2}\right)$, $24.47\left(\mathrm{CH}_{2}\right), 22.56\left(\mathrm{CH}_{2}\right), 14.28$ (Me-30). ESI MS $\mathrm{m} / \mathrm{z}$ (rel.int.): $452[\mathrm{M}]^{+}\left(\mathrm{C}_{30} \mathrm{H}_{60} \mathrm{O}_{2}\right)(68.3)$.

\section{2-Palmityloxybenzyl alcohol (2)}

Elution of the column with chloroform-methanol (97:3, $v / v$ ) yielded greenish semisolid mass of 2 , purified by TLC using chloroform-methanol (3:1, v/v), $997 \mathrm{mg}$ (0.08\% yield), $\mathrm{R}_{\mathrm{f}} 0.6$ $\left(\mathrm{CHCl}_{3}\right)$, UV $\lambda_{\max }(\mathrm{MeOH}): 204,224,287 \mathrm{~nm}(\log \varepsilon$ 4.6, 2.1, 1.8), IR $\lambda \max (\mathrm{KBr}): 3321,2920,2852,1724,1622,1525,1463,1375$, 1247, 1037, $719 \mathrm{~cm}^{-1} ;{ }^{1} \mathrm{H}$ NMR $\left(\mathrm{CDCl}_{3}\right): \delta 7.20(1 \mathrm{H}, \mathrm{d}, J=7.6 \mathrm{~Hz}$, H- 3), 6.24 (1H, dd, $J=8.4,2.3 \mathrm{~Hz}, \mathrm{H}-6), 5.94$ (1H, m, H-5), 5.43 (1H, m, H-4), 3.56 (2H, brs, $\left.\mathrm{H}_{2}-7\right), 2.82\left(2 \mathrm{H}, \mathrm{t}, J=6.5 \mathrm{~Hz}, \mathrm{H}_{2}-2^{\prime}\right)$, $2.38\left(2 \mathrm{H} \mathrm{m}, \mathrm{CH}_{2}\right), 2.13\left(2 \mathrm{H} \mathrm{m}, \mathrm{CH}_{2}\right), 2.03\left(2 \mathrm{H} \mathrm{m}, \mathrm{CH}_{2}\right), 1.68(2 \mathrm{H}$, 
m, $\left.\mathrm{CH}_{2}\right), 1.49\left(2 \mathrm{H} \mathrm{m}, \mathrm{CH}_{2}\right), 1.32\left(18 \mathrm{H}\right.$, brs, $\left.9 \times \mathrm{CH}_{2}\right), 0.96(3 \mathrm{H}, \mathrm{t}$, $\left.J=6.0 \mathrm{~Hz}, \mathrm{Me}-16^{\prime}\right) ;{ }^{13} \mathrm{C}$ NMR $\left(\mathrm{CDCl}_{3}\right): \delta 135.03(\mathrm{C}-1), 142.58$ (C-2), 131.93 (C-3), 130.22 (C-4), 128.24 (C-5), 127.11 (C-6), 71.85 (C-7), 173.12 (C-1'), 37.46 (C-2'), 37.29 (C-3'), 33.98 (C-4'), 33.09 (C-5'), 31.92 (C-6'), 29.69 (C-7'), 29.65 (C-8'), 29.50 (C-9'), 29.35 (C-10'), 29.19 (C-11'), 29.10 (C-12'), 25.58 (C-13'), 24.79 (C-14'), 22.65 (C-15'), 14.11 (C-16'). ESI MS $m / z$ (rel. int.): 362 $[\mathrm{M}]^{+}\left(\mathrm{C}_{23} \mathrm{H}_{38} \mathrm{O}_{3}\right)$ (21.9), 255 (19.1), 239 (15.6), 123 (7.3).

\section{2-O- Diarabinosyl-8"'-geranilanyl salicylate (3)}

Elution of the column with chloroform-methanol (19:1, $v / v)$ afforded a green semisolid mass of $\mathbf{3}$, purified by TLC using acetone-methanol $(3: 1, v / v), \quad 3 \quad \mathrm{~g}(0.24 \%$ yield $), \quad \mathrm{R}_{\mathrm{f}} \quad 0.5$ (chloroform), UV $\lambda_{\max }(\mathrm{MeOH}): 205,227,281 \mathrm{~nm}(\log \varepsilon$ 4.1, 1.8, 1.7). IR $\lambda_{\max }(\mathrm{KBr}): 3482,3390,3292,2926,2854,1721,1635$, $1525,1445,1381,1273,1197,1085,979 \mathrm{~cm}^{-1} .{ }^{1} \mathrm{H}$ NMR $\left(\mathrm{CDCl}_{3}\right)$ : $\delta 7.63(1 \mathrm{H}, \mathrm{dd}, J=3.0,8.8 \mathrm{~Hz}, \mathrm{H}-3), 7.45(1 \mathrm{H}, \mathrm{dd}, J=3.0,9.2 \mathrm{~Hz}$ $\mathrm{H}-6), 7.31(1 \mathrm{H}, \mathrm{m}, \mathrm{H}-5), 7.27(1 \mathrm{H}, \mathrm{m}, \mathrm{H}-4), 5.31(1 \mathrm{H}, \mathrm{d}, J=7.3$ $\left.\mathrm{Hz}, \mathrm{H}-1^{\prime}\right), 4.98\left(1 \mathrm{H}, \mathrm{d}, J=7.1 \mathrm{~Hz}, \mathrm{H}-1^{\prime \prime}\right), 4.38\left(1 \mathrm{H}, \mathrm{m}, \mathrm{H}-2^{\prime}\right), 4.25$ $(1 \mathrm{H}, \mathrm{m}, \mathrm{H}-2 \mathrm{2}), 4.18(1 \mathrm{H}, \mathrm{m}, \mathrm{H}-3), 4.05\left(1 \mathrm{H}, \mathrm{m}, \mathrm{H}-3^{\prime \prime}\right), 4.01(2 \mathrm{H}$, d, $\left.J=6.8 \mathrm{~Hz}, \mathrm{H}_{2}-8^{\prime \prime \prime}\right), 3.85\left(1 \mathrm{H}, \mathrm{m}, \mathrm{H}-4^{\prime}\right), 3.78\left(1 \mathrm{H}, \mathrm{m}, \mathrm{H}-4^{\prime \prime \prime}\right), 3.67$ $\left(2 \mathrm{H}, \mathrm{d}, J=9.1 \mathrm{~Hz}, \mathrm{H}_{2}-5^{\prime}\right), 3.56\left(2 \mathrm{H}, \mathrm{d}, J=9.6 \mathrm{~Hz}, \mathrm{H}_{2}-5 "\right), 2.24(2 \mathrm{H}$, m, $\left.\mathrm{H}_{2}-6^{\prime \prime \prime}\right), 2.11\left(1 \mathrm{H}, \mathrm{m}, \mathrm{H}-7^{\prime \prime}\right), 1.97\left(2 \mathrm{H}, \mathrm{m}, \mathrm{H}_{2}-4^{\prime \prime \prime}\right), 1.74(1 \mathrm{H}, \mathrm{m}$, $\left.\mathrm{H}_{2}-3^{\prime \prime \prime}\right), 1.50$ ( $\left.2 \mathrm{H}, \mathrm{m}, \mathrm{H}_{2}-5^{\prime \prime \prime}\right), 1.19$ ( $\left.2 \mathrm{H}, \mathrm{m}, \mathrm{H}_{2}-2^{\prime \prime \prime}\right), 1.16$ ( $3 \mathrm{H}, \mathrm{d}$, $\left.\mathrm{J}=6.8 \mathrm{~Hz}, \mathrm{Me}-66^{\prime \prime}\right), 0.90\left(3 \mathrm{H}, \mathrm{d}, \mathrm{J}=6.7 \mathrm{~Hz}, \mathrm{Me}-10^{\prime \prime \prime}\right), 0.78 \quad(3 \mathrm{H}$, $\left.\mathrm{t}, J=6.0 \mathrm{~Hz}, \mathrm{Me}-1{ }^{\prime \prime}\right),{ }^{13} \mathrm{C}$ NMR $\left(\mathrm{CDCl}_{3}\right): \delta 132.63(\mathrm{C}-1), 149.11$ (C-2), 128.42 (C-3), 122.56 (C-4), 118.73 (C-5), 119.36 (C-6), 170.38 (C-7), 102.53 (C-1'), 82.49 (C-2'), 71.82 (C-3'), 68.13 (C$\left.4^{\prime}\right), 62.41$ (C-5'), 93.62 (C-1"), 76.19 (C-2"), 71.80 (C-3"), 67.95 (C-4"), 62.24 (C-5"), 14.25 (C-1"'), 27.71 (C-2'"), 47.26 (C-3"'), 29.26 (C-4"'), 29.70 (C-5"'), 31.08 (C-6"'), 42.44 (C-7"'), 66.34 (C8"'), 22.69 (C-9"'), 19.16 (C-10"'). ESI-MS $m / z$ (rel.int.): $542[\mathrm{M}]^{+}$ $\left(\mathrm{C}_{27} \mathrm{H}_{42} \mathrm{O}_{11}\right)$ (8.6), 281 (10.3), 265 (6.3), 149 (29.1), 157 (5.8).

\section{Lignoceryl $O$ - $\beta$ - $D$ - arabinoside (4)}

Elution of the column with chloroform-methanol (93:7, $v / v)$ gave greenish sticky mass of $\mathbf{4}$, purified by TLC using chloroform- methanol $(3: 1, v / v), 5 \mathrm{~g}$ (0.4\% yield), $\mathrm{R}_{\mathrm{f}} 0.4$ (chloroform) UV $\lambda_{\max }(\mathrm{MeOH}): 209 \mathrm{~nm}(\log \varepsilon$ 3.7), IR $\lambda \max$ (KBr): 3435, 3338, 2927, 2854, 1722, 1636, 1440, 1379, 1261, 1207, 975, $722 \mathrm{~cm}^{-1} .{ }^{1} \mathrm{H}$ NMR $\left(\mathrm{CDCl}_{3}\right): \delta 5.31(1 \mathrm{H}, \mathrm{d}, J=7.3 \mathrm{~Hz}$, H-1'), 4.42 (1H, m, H-2'), 4.24 (1H, m, H-3'), 4.05 (1H, m, H-4'), $3.84\left(2 \mathrm{H}, \mathrm{d}, J=6.8 \mathrm{~Hz}, \mathrm{H}-5^{\prime}\right), 2.58\left(2 \mathrm{H}, \mathrm{t}, J=9.6 \mathrm{~Hz}, \mathrm{H}_{2}-2\right), 2.27$ $\left(1 \mathrm{H}, \mathrm{m}, \mathrm{CH}_{2}\right), 2.17\left(2 \mathrm{H}, \mathrm{m}, \mathrm{CH}_{2}\right), 1.60\left(2 \mathrm{H}, \mathrm{m}, \mathrm{CH}_{2}\right), 1.57(2 \mathrm{H}, \mathrm{m}$, $\left.\mathrm{CH}_{2}\right), 1.29\left(6 \mathrm{H}\right.$, brs, $\left.3 \times \mathrm{CH}_{2}\right), 1.24\left(8 \mathrm{H}\right.$, brs, $\left.4 \times \mathrm{CH}_{2}\right), 1.21(10 \mathrm{H}$, brs, $\left.10 \times \mathrm{CH}_{2}\right), 0.84(3 \mathrm{H}, \mathrm{t}, J=7.6 \mathrm{~Hz}, \mathrm{Me}-24) ;{ }^{13} \mathrm{C} \mathrm{NMR}\left(\mathrm{CDCl}_{3}\right)$ $\delta 175.25(\mathrm{C}-1), 55.94(\mathrm{C}-2), 51.95(\mathrm{C}-3), 34.02(\mathrm{C}-4), 31.92(\mathrm{C}-$ 5), 29.70 (C-6), 29.16 (C-7), 29.36 (C-8), 29.28 (C-9), 29.11 (C10), 28.89 (C-11), 31.44 (C-12), 29.36 (C-13), 29.36 (C-14), 29.28 (C-15), 29.21 (C-16), 29.15 (C-17), 29.11 (C-18), 28.89 (C-19), 28.80 (C-20), 27.97(C-21), 24.63 (C-22), 22.70 (C-23), 14.15 (C-24), 103.65 (C-1'), 71.18 (C-2'), $68.47\left(\mathrm{C}-3^{\prime}\right), 66.21$ (C-4'), $63.76\left(\mathrm{C}-5^{\prime}\right)$; ESI-MS $\mathrm{m} / z$ (rel int.): $500[\mathrm{M}]^{+}\left(\mathrm{C}_{29} \mathrm{H}_{56} \mathrm{O}_{6}\right)$ (28.3), 367 (16.1), 351 (33.8), 149 (31.8), 133 (85).

\section{Lignoceryl diarabinoside (5)}

Elution of the column with chloroform-methanol (9:1, $v / v)$ gave dark brown sticky mass of $\mathbf{5}$, purified by TLC using chloroform-methanol $(9: 1, v / v), 5 \mathrm{~g}(0.4 \%$ yield $), \mathrm{R}_{\mathrm{f}} 0.3$ (chloroform); UV $\lambda_{\max }(\mathrm{MeOH}): 207 \mathrm{~nm}(\log \varepsilon$ 3.1), IR $\lambda \max$ (KBr): 3455, 3390, 3290, 2926, 2854, 1721, 1440, 1635, 1458, $1259,1039,722 \mathrm{~cm}^{-1} .{ }^{1} \mathrm{H}$ NMR $\left(\mathrm{CDCl}_{3}\right): \delta 5.31(1 \mathrm{H}, \mathrm{d}, J=7.5 \mathrm{~Hz}$, $\left.\mathrm{H}-1^{\prime}\right), 5.28\left(1 \mathrm{H}, \mathrm{d}, J=7.1 \mathrm{H}-1^{\prime \prime}\right), 4.30\left(1 \mathrm{H}, \mathrm{m}, \mathrm{H}-2^{\prime}\right), 4.08(1 \mathrm{H}, \mathrm{m}$, H-2"), 3.90 (1H, m, H-3'), 3.84 (1H, m, H-3"), 3.79 (1H, m, H-4'), $3.76(1 \mathrm{H}, \mathrm{m}, \mathrm{H}-4 "), 3.63\left(2 \mathrm{H}, \mathrm{d}, J=5.6 \mathrm{~Hz}, \mathrm{H}_{2}-5^{\prime}\right), 3.57(2 \mathrm{H}, \mathrm{d}$, $\left.J=6.6 \mathrm{~Hz}, \mathrm{H}_{2}-5 "\right), 2.79\left(2 \mathrm{H}, \mathrm{t}, J=7.3 \mathrm{~Hz}, \mathrm{H}_{2}-2\right), 2.57\left(2 \mathrm{H}, \mathrm{m}, \mathrm{CH}_{2}\right)$, $2.26\left(2 \mathrm{H}, \mathrm{m}, \mathrm{CH}_{2}\right), 2.23\left(2 \mathrm{H}, \mathrm{m}, \mathrm{CH}_{2}\right), 2.16\left(2 \mathrm{H}, \mathrm{m}, \mathrm{CH}_{2}\right), 2.01$ $\left(2 \mathrm{H}, \mathrm{m}, \mathrm{CH}_{2}\right), 1.60\left(2 \mathrm{H}, \mathrm{m}, \mathrm{CH}_{2}\right), 1.53\left(2 \mathrm{H}, \mathrm{m}, \mathrm{CH}_{2}\right), 1.29(2 \mathrm{H}, \mathrm{m}$, $\left.\mathrm{CH}_{2}\right), 1.24\left(6 \mathrm{H}\right.$, brs, $\left.3 \times \mathrm{CH}_{2}\right), 1.21\left(20 \mathrm{H}\right.$, brs, $\left.10 \times \mathrm{CH}_{2}\right), 0.83$ $(3 \mathrm{H}, \mathrm{t}, J=7.2 \mathrm{~Hz}, \mathrm{Me}-24) .{ }^{13} \mathrm{C} \mathrm{NMR}\left(\mathrm{CDCl}_{3}\right): \delta 171.56(\mathrm{C}-1)$, 42.91 (C-2), 34.01 (C-3), 31.91 (C-4), 31.43 (C-5), 30.18 (C-6), 29.69 (C-7), 29.69 (C-8), 29.69 (C-9), 29.69 (C-10), 29.69 (C11), 29.51 (C-12), 29.48 (C-13), 29.35 (C-14), 29.14 (C-15), 29.47 (C-16), 29.85 (C-17), 29.96 (C-18), 27.73 (C-19), 27.15 (C-20), 25.32(C-21), 24.77 (C-22), 22.68 (C-23), 14.10 (C-24), $101.93\left(\mathrm{C}-1^{\prime}\right), 80.21\left(\mathrm{C}-2^{\prime}\right), 74.55\left(\mathrm{C}-3^{\prime}\right), 65.61\left(\mathrm{C}-4^{\prime}\right), 63.16(\mathrm{C}-$ 5'), 102.48 (C-1"), 72.34 (C-2"), 68.42 (C-3"), 64.35 (C-4"), 62.27 (C-5"). ESI-MS m/z (rel.int.): $632[\mathrm{M}]^{+}\left(\mathrm{C}_{34} \mathrm{H}_{64} \mathrm{O}_{10}\right)$ (38.4), 367 (10.8), 351 (53.1), 281 (32.3), 265 (11.2), 149 (2.7).

\section{Trihydroxynaphthyl tetraglycosidic stearate (6)}

Elution of the column with chloroform-methanol (3:1, $v / v$ ) furnished red semisolid mass of $\mathbf{6}$, purified by TLC using methanol, $7.91 \mathrm{~g}\left(0.64 \%\right.$ yield); $\mathrm{R}_{\mathrm{f}} 0.2$ (chloroform-methanol 3:1); UV $\lambda \max (\mathrm{MeOH}): 207,285 \mathrm{~nm}$. $(\log \varepsilon$ 4.1, 1.3). IR $\lambda \max (\mathrm{KBr})$ : 3490, 3392, 3281, 2927, 2858, 1721, 1664, 1630, 1525, 1458, 1381, 1263, 1070, $722 \mathrm{~cm}^{-1} .{ }^{1} \mathrm{H}$ NMR ( DMSO-d $\left.)_{6}\right): \delta 7.03(1 \mathrm{H}, \mathrm{d}$, $J=2.2 \mathrm{~Hz}, \mathrm{H}-1), 6.75(1 \mathrm{H}, \mathrm{dd}, J=2.2,8.0 \mathrm{~Hz}, \mathrm{H}-3), 6.41(1 \mathrm{H}, \mathrm{d}$, $J=8.0 \mathrm{~Hz}, \mathrm{H}-4), 5.93(1 \mathrm{H}, \mathrm{s}, \mathrm{H}-5), 5.91(1 \mathrm{H}, \mathrm{s}, \mathrm{H}-8), 5.40(1 \mathrm{H}, \mathrm{d}$, $J=7.3 \mathrm{~Hz}, \mathrm{H}-1 \mathrm{a}), 3.97$ (1H, m, H-5a), 3.73, (1H, m, H-2a), 3.58 (1H, m, H-3a), 3.50 (1H, m, H-4a), 3.29 (2H, d, J=4.8 Hz, H $\left.{ }_{2}-6\right)$, $5.28(1 \mathrm{H}, \mathrm{d}, J=7.1 \mathrm{~Hz}, \mathrm{H}-1 \mathrm{~b}), 3.95$ (1H, m, H-5b), 3.67(1H, m, H2b), 3.56 (1H, m, H-3b), 3.48 (1H, m, H-4b), 3.24 (2H, d, J=5.6 $\left.\mathrm{Hz}, \mathrm{H}_{2}-6 \mathrm{~b}\right), 5.20$ (1H, d, J=7.2 Hz, H-1c), 4.02 (1H, m, H-2c), 3.91(1H, m, H-5c), 3.54 (1H, m, H-3c), 3.45 (1H, m, H-4c), 5.10 (1H, d, J=7.0 Hz, H-1d), 4.33 (1H, m, H-2d), 3.90 (1H, m, H-5d), 3.51 (1H, m, H-3d), 3.38 (1H, m, H-4d), 2.59 (2H, t, J=7.2 Hz, H $\left.2^{\prime}\right), 2.18\left(2 \mathrm{H}, \mathrm{m}, \mathrm{CH}_{2}\right), 2.08\left(2 \mathrm{H}, \mathrm{m}, \mathrm{CH}_{2}\right), 1.88\left(2 \mathrm{H}, \mathrm{m}, \mathrm{CH}_{2}\right), 1.40$ ( $2 \mathrm{H}, \mathrm{m}, \mathrm{CH}_{2}$ ), 1.15 (4H, brs, $2 \times \mathrm{CH}_{2}$ ), 1.14 (4H, brs, $2 \times \mathrm{CH}_{2}$ ), $1.12\left(14 \mathrm{H}\right.$, brs, $\left.7 \times \mathrm{CH}_{2}\right), 0.72(3 \mathrm{H}, \mathrm{t}, J=6.2 \mathrm{~Hz}, \mathrm{Me}-18$ '); ${ }^{13} \mathrm{C}$ NMR (DMSO-d ${ }_{6}$ ): $\delta 146.16$ (C-1), 151.03 (C-2), 128.24 (C-3), 122.32 (C-4), 142.31 (C-5), 148.56 (C-6), 147.14 (C-7), 140.06 (C-8), 131.83 (C-9), 144.41 (C-10), 108.89 (C-1a), 74.08 (C-2a), 74.39 (C-3a), 70.20 (C-4a), 77.13 (C-5a), 63.60 (C-6a), 108.51 (C1b), 73.31 (C-2b), 74.39 (C-3b), 69.64 (C-4b), 77.13 (C-5b), 63.60 (C-6b), 106.50 (C-1c), 85.01 (C-2c), 72.13 (C-3c), 69.53 (C-4c), 76.28 (C-5c), 176.18 (C-6c), 101.98 (C-1d), 82.67 (C-2d), 74.39 (C-3d), 69.39 (C-4d), 75.03 (C-5d), 177.48 (C-6d), 174.02 (C-1'), 55.40 (C-2'), 42.57 (C-3'), 34.12 (C-4'), 31.79 (C-5'), 30.79 (C-6'), 
29.60 (C-7'), 29.60 (C-8'), 29.60 (C-9'), 29.41 (C-10'), 29.37 (C11'), 29.19 (C-12'), 29.11 (C-13'), 27.13 (C-14'), 25.62 (C-15'), 24.87 (C-16'), 22.69 (C-17'), 14.33 (C-18'). ESI MS m/z (rel.int.): $1118[\mathrm{M}]^{+}\left(\mathrm{C}_{52} \mathrm{H}_{78} \mathrm{O}_{26}\right)$ (4.5), 675 (12.8), 619 (13.1), 499 (12.2), 443 (31.7), 267 (17.5), 175 (13.2).

\section{Salicylic acid 2-O- $\beta$-tetra-arabinosyl tetra methyl hexadecanoate $(7)$}

Elution of the column with chloroform-methanol $(3: 1$, $v / v$ ) furnished brown colour semisolid mass of 7 , purified by TLC using chloroform-methanol $(1: 1, v / v), 10 \mathrm{~g}$ (0.8\% yield); $\mathrm{R}_{\mathrm{f}} 0.2$ (chloroform), UV $\lambda \max (\mathrm{MeOH}): 207,257,275 \mathrm{~nm}$; $(\log \varepsilon$ 4.6, 2.8, 2.1). IR $v_{\max }(\mathrm{KBr}): 3505,3431,3255,2933,2870,1723$, 1690, 1635, 1597, 1440, 1394, 1263, $1066 \mathrm{~cm}^{-1}$. ${ }^{1} \mathrm{H}$ NMR (DMSO$\left.\mathrm{d}_{6}\right): \delta 7.66(1 \mathrm{H}, \mathrm{dd}, J=2.8,8.5 \mathrm{~Hz}, \mathrm{H}-3), 7.45(1 \mathrm{H}, \mathrm{dd}, J=2.3,8.5$ Hz, H-6), 7.35 (1H, m, H-5), 7.30 (1H, m, H-4), 5.21 (1H, d, J=7.2 Hz, H-1a), 5.10 (1H, d, J=7.1 Hz, H-1b), $5.01(2 \mathrm{H}, \mathrm{d}, \mathrm{J}=7.3 \mathrm{~Hz}, \mathrm{H}-$ 1c, H-1d), 4.20 (1H, m, H-2d), 4.03 (1H, m, H-2a), 3.97 (1H, m, H-2b), 3.92 (1H, m,H-2c), 3.74 (1H, m, H-3a), 3.70 (1H, m, H3b), 3.68 (1H, m, H-3c), 3.65 (1H, m, H-3d), 3.62 (2H, m, H-4a, H-4b), 3.60 (2H, m, H-4c, H-4d), 3.58 (2H, d, J=6.0 Hz, $\left.\mathrm{H}_{2}-5 \mathrm{a}\right)$, $3.55\left(2 \mathrm{H}, \mathrm{d}, J=11.2 \mathrm{~Hz}, \mathrm{H}_{2}-5 \mathrm{~b}\right), 3.52\left(2 \mathrm{H}, \mathrm{d}, J=7.2 \mathrm{~Hz}, \mathrm{H}_{2}-5 \mathrm{c}\right)$, $3.49\left(2 \mathrm{H}, \mathrm{d}, J=6.0 \mathrm{~Hz}, \mathrm{H}_{2}-5 \mathrm{~d}\right), 2.28\left(1 \mathrm{H}, \mathrm{m}, \mathrm{H}-2^{\prime}\right), 2.20(1 \mathrm{H}, \mathrm{m}, \mathrm{H}-$ 6'), 1.91 (1H, m, H-10'), 1.88 (1H, m, H-14'), 1.49 (2H, m, $\left.\mathrm{H}_{2}-3^{\prime}\right)$, $1.47\left(2 \mathrm{H}, \mathrm{m}, \mathrm{H}_{2}-4^{\prime}\right), 1.20$ (2H, m, H $\left.{ }_{2}^{-5}, \mathrm{H}_{2}-7^{\prime}\right), 1.16$ (12H, brs, $6 \mathrm{x}$ $\left.\mathrm{CH}_{2}\right), 0.94(3 \mathrm{H}, \mathrm{d}, J=6.0 \mathrm{~Hz}, \mathrm{Me}-17$ '), $0.90(3 \mathrm{H}, \mathrm{d}, J=6.0 \mathrm{~Hz}, \mathrm{Me}-$ $\left.19^{\prime}\right), 0.87$ (3H, d, J=7.2 Hz, Me-18'), 0.84 (3H, d, $J=6.8 \mathrm{~Hz}, \mathrm{Me}-$ 20'), $0.79\left(3 \mathrm{H}, \mathrm{d}, J=6.8 \mathrm{~Hz}, \mathrm{Me}-16^{\prime}\right) ;{ }^{13} \mathrm{C} \mathrm{NMR}\left(\mathrm{DMSO}-\mathrm{d}_{6}\right) ; \delta$ 141.98 (C-1), 153.21 (C-2), 131.45 (C-3), 127.67 (C-4), 127.88 (C-5), 128.70 (C-6), 180.36 (C-7), 103.65 (C-1a), 76.73 (C-2a), 73.70 (C-3a), 70.09 (C-4a), 62.67 (C-5a), 103.49 (C-1b), 75.22 (C2b), 72.81 (C-3b), 70.29 (C-4b), 62.91 (C-5b), 101.74 (C-1c), 74.77 (C-2c), 71.91 (C-3c), 69.66 (C-4c), 63.15 (C-5c), 98.04 (C1d), 83.15 (C-2d), 71.73 (C-3d), 69.02 (C-4d), 63.23 (C-5d), 171.63 (C-1'), 56.59 (C-2'), 31.60 (C-3'), 29.39 (C-4'), 29.13 (C$\left.5^{\prime}\right), 39.99$ (C-6'), 24.61 (C-7'), 24.25 (C-8'), 24.38 (C-9'), 36.46 (C10'), 22.31 (C-11'), 21.88 (C-12'), 21.55 (C-13'), 33.83 (C-14'), 20.91 (C-15'), 10.99 (C-16'), 17.71 (C-17'), 16.82 (C-18'), 14.23 (C-19'), 13.22 (C-20'). ESI MS m/z (rel.int.): $960[\mathrm{M}]^{+}\left(\mathrm{C}_{47} \mathrm{H}_{76} \mathrm{O}_{20}\right)$ (2.1), 665 (6.8), 427 (14.5), 401 (12.3), 295 (18.2), 137 (11.8).

\section{RESULTS AND DISCUSSION}

Compound $\mathbf{1}$ was a known fatty acid identified as $n$ triacontanoic acid (melissic acid) (Chibnall et al., 1933).

Compound 2, designated as 2-palmityloxybenzyl alcohol, showed characteristic IR absorption bands for hydroxyl group $\left(3321 \mathrm{~cm}^{-1}\right)$, ester function $\left(1724 \mathrm{~cm}^{-1}\right)$, aromatic ring $(1622,1525$ $\left.\mathrm{cm}^{-1}\right)$ and long aliphatic chain $\left(719 \mathrm{~cm}^{-1}\right)$. On the basis of mass and ${ }^{13} \mathrm{C}$ NMR spectra the molecular ion peak of $\mathbf{2}$ was determined at $\mathrm{m} / \mathrm{z} 362$ consisting to the molecular formula of a phenyl alcoholic ester $\mathrm{C}_{23} \mathrm{H}_{38} \mathrm{O}_{3}$. The ion fragments arising at $\mathrm{m} / z \quad 239$ $\left[\mathrm{CH}_{3}\left(\mathrm{CH}_{2}\right){ }_{14} \mathrm{CO}\right]^{+}, \mathrm{m} / z, 255\left[\mathrm{CH}_{3}\left(\mathrm{CH}_{2}\right)_{14} \mathrm{COO}\right]^{+}$and 123 [M-239; $\left.\mathrm{C}_{6} \mathrm{H}_{4} \mathrm{OCH}_{2} \mathrm{OH}\right]$ indicated that palmitic acid was esterified with a hydroxyl benzyl alcohol. The ${ }^{1} \mathrm{H}$ NMR spectrum of 2 showed a one-proton doublet at $\delta 7.20(J=7.6 \mathrm{~Hz})$, a one-proton double doublet at $6.24(J=2.3,8.4 \mathrm{~Hz})$, and two one-proton multiplets at $\delta$ 5.94 and 5.43 assigned to aromatic $\mathrm{H}-3, \mathrm{H}-6, \mathrm{H}-5$ and $\mathrm{H}-4$ protons, respectively. A two-proton broad singlet at $\delta 3.56$ was ascribed to hydroxylmethylene $\mathrm{H}_{2}-7$ protons. A two-proton triplet at $\delta 2.82$ $(J=6.5 \mathrm{~Hz})$, four two- proton multiplets between $\delta 2.38-1.49$ and a broad singlet at $\delta 1.32(18 \mathrm{H})$ were associated with the methylene protons of the acyl chain. A three-proton triplet at $\delta 0.96(J=6.0$ $\mathrm{Hz}$ ) was accounted to C-16' primary methyl protons. The ${ }^{13} \mathrm{C}$ NMR spectrum of 2 showed signals for aromatic carbons between $\delta$ 135.03-127.11, benzyl methylene carbon at $\delta 71.85$ (C-7), ester carbon at $\delta 173.12\left(\mathrm{C}-1^{\prime}\right)$ and remaining carbon signals are resonated between $\delta$ 37.46-14.11. Acid hydrolysis of 2 yielded pamitic acid, co-TLC comparable. On the basis of above discussion the structure of compound $\mathbf{2}$ was formulated as 2 hexadecanoxybenzyl alcohol. This is a new aromatic alcohol ester. Compound 3, named 2-O-diarabinosyl-8"'-geranilanyl salicylate, was obtained as a green amorphous mass from chloroformmethanol $(19: 1 \quad v / v)$ eluants. It gave positive tests for carbohydrates and displayed characteristic IR absorption bands for hydroxyl groups $\left(3482,3390,3292 \mathrm{~cm}^{-1}\right)$, ester function $(1721 \mathrm{~cm}$ $\left.{ }^{1}\right)$ and aromatic ring $\left(1635,1525,1085 \mathrm{~cm}^{-1}\right)$. The molecular ion peak of 3 was determined at $m / z, 542$ on the basis of mass and ${ }^{13} \mathrm{C}$ NMR spectra corresponding to a molecular formula of a phenolic acid glycosidic ester $\mathrm{C}_{27} \mathrm{H}_{42} \mathrm{O}_{11}$. The ion peaks arising at $m / z, 149$ $\left[\mathrm{C}_{5} \mathrm{H}_{9} \mathrm{O}_{5}\right]^{+}, 265 \quad\left[\mathrm{C}_{5} \mathrm{H}_{9} \mathrm{O}_{5}-\mathrm{C}_{5} \mathrm{H}_{8} \mathrm{O}_{3}\right]^{+}$and $281 \quad\left[\mathrm{C}_{5} \mathrm{H}_{9} \mathrm{O}_{5}-\mathrm{C}_{5} \mathrm{H}_{8} \mathrm{O}_{4}\right]^{+}$ suggested that two $\mathrm{C}-5$ sugar units were linked to phenyl group. An ion peak formed at $m / z \quad 157\left[\mathrm{C}_{10} \mathrm{H}_{21} \mathrm{O}\right]^{+}$suggested that a $\mathrm{C}-10$ alcohol was esterified with the aromatic acid. The ${ }^{1} \mathrm{H}$ NMR spectrum of 3 exhibited two one-proton double doublets at $\delta 7.63$ $(J=3.0,8.8 \mathrm{~Hz})$ and $7.45(J=3.0,9.0 \mathrm{~Hz})$ and two one-proton multiplets at $\delta 7.31$ and 7.27 assigned to aromatic $\mathrm{H}-3, \mathrm{H}-6, \mathrm{H}-5$ and $\mathrm{H}-4$ protons, respectively. Two one-proton doublets at $\delta 5.31$ $(J=7.3 \mathrm{~Hz})$ and $4.98(J=7.1 \mathrm{~Hz})$ were ascribed to anomeric $\mathrm{H}-1$ and $\mathrm{H}-1$ " protons, respectively. The other sugar protons appeared between $\delta 4.38-3.56$. A two-proton doublet at $\delta 4.01(J=6.8 \mathrm{~Hz})$ was accounted to oxygenated methylene $\mathrm{H}_{2}-8$ "' protons and its presence in the deshielded region indicated the location of the ester group at C-8"'. Two three-proton doublets at $\delta 1.16(J=6.8 \mathrm{~Hz})$ and $0.90(J=6.7 \mathrm{~Hz})$ and a three-proton triplet at $\delta 0.78(J=6.0 \mathrm{~Hz})$ were associated correspondingly with the secondary $\mathrm{C}-9$ "' and $\mathrm{C}$ 10 "' and primary C-1"' methyl protons of monoterpenic unit. The remaining methylene and methine proton resonated from $\delta 2.24$ to 1.19. The ${ }^{13} \mathrm{C}$ NMR spectrum of $\mathbf{3}$ showed signals for aromatic carbons between $\delta 149.11-119.36$, ester group at $\delta 170.38$ (C-7), anomeric carbons at $\delta 102.53\left(\mathrm{C}-1^{\prime}\right)$ and $\delta 93.62$ (C-1"), while remaining sugar protons appeared between $\delta$ 82.49-62.24. Downfield signal of terpenic carbon at $\delta 66.34$ (C-8"') due to adjacent to ester group, remaining methine and methylene carbon are appeared between $\delta$ 27.71-31.08 and methyl carbons at $\delta$ 14.25 (C-1"') and $\delta 19.16$ (C-10'"). On the basis of aforementioned evidences the structure of 3 was elucidated as $2-O-\beta-D$ arabinopyranosyloxy-2'- $O-\beta-D$-arabinopyranosyl-(8"'-geranilanyl) benzoate. This is a new phenolic glycoside. Compound $\mathbf{4}$, 
designated as lignoceryl $O-\beta-D$ - arabinoside, gave positive tests for glycosides and displayed characteristic IR absorption bands for hydroxyl groups (3435 and $\left.3338 \mathrm{~cm}^{-1}\right)$, ester function $\left(1722 \mathrm{~cm}^{-1}\right)$, and long chain aliphatic hydrocarbon $\left(722 \mathrm{~cm}^{-1}\right)$. On the basis of mass and ${ }^{13} \mathrm{C}$ NMR spectra the molecular ion peak of 4 was established at $\mathrm{m} / \mathrm{z}, 500$ corresponding to a molecular formula of an acyl glycoside, $\mathrm{C}_{29} \mathrm{H}_{56} \mathrm{O}_{6}$. The ion peaks arising at $\mathrm{m} / \mathrm{z}, 133$ $\left[\mathrm{C}_{5} \mathrm{H}_{9} \mathrm{O}_{4}\right]^{+}, 149\left[\mathrm{C}_{5} \mathrm{H}_{9} \mathrm{O}_{5}\right]^{+}, 367\left[\mathrm{M}-133, \mathrm{CH}_{3}\left(\mathrm{CH}_{2}\right)_{22} \mathrm{COO}\right]^{+}$and 351 [M-149, $\left.\mathrm{CH}_{3}\left(\mathrm{CH}_{2}\right)_{22} \mathrm{CO}\right]^{+}$suggested that a $\mathrm{C}_{5}$ sugar unit was esterified with a $\mathrm{C}_{24}$ fatty acid. The ${ }^{1} \mathrm{H}$ NMR spectrum of 4 exhibited a one-proton doublet at $\delta 5.31(J=7.3 \mathrm{~Hz})$ assigned to anomeric $\mathrm{H}-1^{\prime}$ protons. Three one-protons multiplets between $\delta$ 4.42-4.05 were ascribed to sugar carbinol protons, a two-protons doublets at $\delta 3.84(J=8.5 \mathrm{~Hz})$ was accounted to oxygenated methylene $\mathrm{H}_{2}-5^{\prime}$ protons. A two-proton triplets at $\delta 2.58(J=9.6$ $\mathrm{Hz}$ ) was due to methylene $\mathrm{H}_{2}-2$ proton adjacent to the ester group, and remaining methylene protons appeared between $\delta 2.27-1.21$. A three-proton triplet at $\delta 0.84(J=7.6 \mathrm{~Hz})$ was associated with the terminal methyl $\mathrm{H}_{3}-24$ protons. The ${ }^{13} \mathrm{C}$ NMR spectrum of 4 exhibited signals for the ester carbon at $\delta 175.25$ (C-1), anomeric carbon at $\delta 103.65\left(\mathrm{C}-1^{\prime}\right)$, other sugar carbons in the range from $\delta$ 71.18 to 63.76 , methylene carbons between $\delta 55.94-22.70$ and methy1 carbon at 14.15 (C-24). Acid hydrolysis of 4 yielded lignoceric acid and D-arabinose, co-TLC comparable. On the basis of the above mentioned discussion the structure of $\mathbf{4}$ has been elucidated as $n$-tetracosanoyl- $O-\beta-D$-arabinopyranoside. This is new acyl glycoside.

Compound 5, designated as lignoceryl diarabinoside, was a dark brown semisolid mass. It gave positive tests for glycosides and displayed characteristic IR absorption bands for hydroxyl groups $\left(3455,3390,3290 \mathrm{~cm}^{-1}\right)$, ester function $\left(1721 \mathrm{~cm}^{-1}\right)$, and long chain aliphatic hydrocarbon $\left(722 \mathrm{~cm}^{-1}\right)$. The molecular ion peak arising at $\mathrm{m} / \mathrm{z}, 632$ consistent to molecular formula of acyl diglycoside, $\mathrm{C}_{34} \mathrm{H}_{64} \mathrm{O}_{10}$. The ion fragments at $m / z, 149\left[\mathrm{C}_{5} \mathrm{H}_{9} \mathrm{O}_{5}\right]^{+}$, $265\left[\mathrm{C}_{5} \mathrm{H}_{9} \mathrm{O}_{5}-\mathrm{C}_{5} \mathrm{H}_{8} \mathrm{O}_{3}\right]^{+}$and $281\left[\mathrm{C}_{5} \mathrm{H}_{9} \mathrm{O}_{5}-\mathrm{C}_{5} \mathrm{H}_{8} \mathrm{O}_{4}\right]^{+}, 367[\mathrm{M}-265$, $\left.\mathrm{CH}_{3}\left(\mathrm{CH}_{2}\right)_{22} \mathrm{COO}\right]^{+}$and 351 [M-281, $\left.\mathrm{CH}_{3}\left(\mathrm{CH}_{2}\right)_{22} \mathrm{CO}\right]^{+}$suggested that behenic acid was esterified with a diarabinose unit. The ${ }^{1} \mathrm{H}$ NMR spectrum of $\mathbf{5}$ exhibited two one-proton doublets at $\delta 5.31$ $(J=7.5 \mathrm{~Hz})$ and $\delta 5.28(J=7.1 \mathrm{~Hz})$ assigned to anomeric $\mathrm{H}-1^{\prime}$ and $\mathrm{H}-1^{\prime \prime}$ protons, respectively. Six one-protons multiplets appeared between $\delta 4.30$ - 3.76 each assigned for carbinol protons, two twoproton doublets at $\delta 3.63(J=5.6 \mathrm{~Hz})$ and $\delta 3.57(J=6.6 \mathrm{~Hz})$ were accounted to oxygenated methylene $\mathrm{H}_{2}-5^{\prime}$ and $\mathrm{H}_{2}-5^{\prime \prime}$ protons, respectively. A two-proton triplet at $\delta 2.79(J=7.3 \mathrm{~Hz})$ was ascribed to methylene $\mathrm{H}_{2}-2$ proton nearby to the ester function, and the remaining methylene protons appeared between $\delta 2.57-1.21$. A three-proton triplet at $\delta 0.83(J=7.2 \mathrm{~Hz})$ was due to terminal methyl $\mathrm{H}_{3}-24$ protons. The ${ }^{13} \mathrm{C}$ NMR spectrum of 5 exhibited signals for the ester carbon at $\delta 171.56(\mathrm{C}-1)$, anomeric carbons at $\delta 101.93\left(\mathrm{C}-1^{\prime}\right)$ and $\delta 102.48\left(\mathrm{C}-1^{\prime \prime}\right)$, other sugar carbons in the range from $\delta 80.21$ to 62.27 , methylene carbons between $\delta 42.91$ 22.68 and methyl carbon at $14.06(\mathrm{C}-24)$. The presence of ${ }^{1} \mathrm{H}$ NMR signal for $\mathrm{H}-2^{\prime}$ in the deshielded region at $\delta 4.30$ and ${ }^{13} \mathrm{C}$ NMR signals for $\mathrm{C}-2^{\prime}$ at $\delta 80.21$ suggested the attachment of another sugar at $\left(2^{\prime} \rightarrow 1^{\prime \prime}\right)$ linkage. Acid hydrolysis of 5 yielded lignoceric acid and $D$-arabinose, co-TLC comparable. On the basis of these evidences the structure of $\mathbf{5}$ has been elucidated as $n$ tetracosanoyl- $O-\beta-D-\quad$ arabinopyranosyl- $\left(2^{\prime} \rightarrow 1^{\prime \prime}\right)-O-\beta-D-$ arabinopyranoside. This is a new fatty ester diarabinoside.

Compound 6, named as trihydroxynaphthyl tetraglycosidic stearate, was obtained as a red sticky semisolid mass. It responded glycosidic tests positively and showed IR absorption bands for hydroxyl groups (3490, 3392, $\left.3281 \mathrm{~cm}^{-1}\right)$, ester group $\left(1721 \mathrm{~cm}^{-1}\right)$, aromatic ring $\left(1525,1458,1070 \mathrm{~cm}^{-1}\right)$ and long chain aliphatic hydrocarbon $\left(722 \mathrm{~cm}^{-1}\right)$. On the basis of mass and ${ }^{13} \mathrm{C}$ NMR spectral data, the molecular ion peak of 6 was established at $\mathrm{m} / \mathrm{z} 1118$ corresponding to the molecular formula of a trihydroxynaphthyl tetraglycosidic ester $\left(\mathrm{C}_{52} \mathrm{H}_{78} \mathrm{O}_{26}\right)$. An ion peak generating at $m / z 175\left[\mathrm{O}-\mathrm{C}_{1 \mathrm{a}} \text { fission, } \mathrm{C}_{10} \mathrm{H}_{7}(\mathrm{O})_{3}\right]^{+}$suggested the existence of a trihydroxynaphthyl unit in the molecule. The ion fragments arising at $m / z 267 \quad\left[\mathrm{CH}_{3}\left(\mathrm{CH}_{2}\right)_{16} \mathrm{CO}\right]^{+}$indicated the attachment of a $\mathrm{C}_{18}$ acyl unit in the compound. The ion peaks produced at $m / z \quad 443 \quad\left[\mathrm{CH}_{3}\left(\mathrm{CH}_{2}\right)_{16} \mathrm{CO}-\mathrm{C}_{5} \mathrm{H}_{8} \mathrm{O}_{4} \mathrm{COOH}\right]^{+}, \quad 619$ $\left[\mathrm{CH}_{3}\left(\mathrm{CH}_{2}\right)_{16} \mathrm{CO}-\left(\mathrm{C}_{5} \mathrm{H}_{8} \mathrm{O}_{4} \mathrm{COOH}\right)_{2}\right]^{+}, 675[\mathrm{M}-443]^{+}$and $499[\mathrm{M}-$ $619]^{+}$supported the presence of stearyl substituted glycosidic acid units at the terminal position linked to the remaining glycosidic chain. The ${ }^{1} \mathrm{H}$ NMR spectra of 6 exhibited two one- proton doublets at $\delta 7.03(\mathrm{~J}=2.2 \mathrm{~Hz})$ and $6.41(\mathrm{~J}=8.0 \mathrm{~Hz})$, a one-proton double doublet at $\delta 6.75(\mathrm{~J}=2.2,8.0 \mathrm{~Hz})$, and two one-proton singlet at $\delta 5.93$ and 5.91 assigned, respectively, to aromatic metacoupled $\mathrm{H}-1$ adjacent to the glycoside linked phenolic group, ortho-coupled H-4, meta, ortho-coupled H-3 and para-coupled H-5 and H-8 protons. Four one-proton doublets at $\delta 5.40(J=7.3 \mathrm{~Hz})$, $5.28(J=7.1 \mathrm{~Hz}), 5.20(J=7.2 \mathrm{~Hz})$ and $5.10(J=7.0 \mathrm{~Hz})$ were assigned to sugar anomeric $\mathrm{H}-1 \mathrm{a}, 1 \mathrm{~b}, 1 \mathrm{c}$ and $1 \mathrm{~d}$ protons, respectively. The other sugar protons resonated from $\delta 4.33$ to 3.18. A two-proton triplet at $\delta 2.59(J=7.2 \mathrm{~Hz})$ was ascribed to methylene $\mathrm{H}_{2}-2$ ' protons adjacent to the ester group. A threeproton triplet at $\delta 0.72(\mathrm{~J}=6.2 \mathrm{~Hz})$ was due to terminal C-18' primary methyl protons. The remaining methylene protons appeared between $\delta$ 2.18-1.12. The ${ }^{13} \mathrm{C}$ NMR spectrum of 6 displayed signals for naphthalenic carbons between $\delta 151.03$ to 122.32 , ester carbon at $\delta 174.02\left(\mathrm{C}-1^{\prime}\right)$, anomeric carbons at $\delta$ 108.89 (C-1a), 108.51 (C-1b), 106.50 (C-1c) and 101.98 (C-1d), carboxylic carbons at $\delta 176.18$ (C-6c) and $\delta 177.48$ (C-6d), remaining sugar carbons resonated between $\delta 82.67$ to 63.60, methylene carbons from $\delta 55.40$ to 22.69 , and terminal methyl carbon at $\delta 14.33$ (C-18'). The presence of downfield signals of two oxygenated methylene protons as doublets at $\delta 3.29(\mathrm{~J}=4.8$ $\mathrm{Hz}$ ) for $\mathrm{H}_{2}-6$ a and at $\delta 3.24\left(J=5.6 \mathrm{~Hz}\right.$ ) for $\mathrm{H}_{2}-6 \mathrm{~b}$ in the ${ }^{1} \mathrm{H}$ NMR spectrum and carbon signals $\delta 63.60$ (C-6a, C-6b) in the ${ }^{13} \mathrm{C}$ NMR spectrum suggested the $(6 a \rightarrow 1 b)$ and $(6 b \rightarrow 1 c)$ linkages between two sugar units, and the existence of two downfield signals as multiplets at $\delta 4.02(\mathrm{H}-2 \mathrm{c})$ and $\delta 4.33(\mathrm{H}-2 \mathrm{~d})$ and the carbon signals at $\delta 85.01(\mathrm{C}-2 \mathrm{c})$ and $\delta 82.67(\mathrm{C}-2 \mathrm{~d})$ suggested that $(2 \mathrm{c} \rightarrow 1 \mathrm{~d})$ and $\left(2 \mathrm{~d} \rightarrow 1^{\prime}\right)$ linkages of two sugar units and aliphatic chain. On the basis of above discussion the compound 6 is structurally elucidated as 2,6,7- trihydroxynaphthyl-2- $O-\beta-D$ - 
glucopyranosyl-(6a $\rightarrow 1 \mathrm{~b})-O-\beta-D$-glucopyranosyl-( $6 \mathrm{~b} \rightarrow 1 \mathrm{c})-O-\beta-D$ glucuranopyranosyl-( $2 \mathrm{c} \rightarrow 1 \mathrm{~d})-O-\beta-D$-glucuranopyranosyl-2d- $n$ octadecanoate. This is new naphthol tetraglycosidic ester.

Compound 7, named salicylic acid 2-O- $\beta$-tetra-arabinosyl tetra methyl hexadecanoate, responded glycosidic tests positively and showed IR absorption bands for hydroxyl groups (3505, 3431, $\left.\mathrm{cm}^{-1}\right)$, carboxylic group $\left(3255,1690 \mathrm{~cm}^{-1}\right)$, ester group $\left(1723 \mathrm{~cm}^{-}\right.$ $\left.{ }^{1}\right)$, and aromatic ring $\left(1597,1066 \mathrm{~cm}^{-1}\right)$. On the basis of mass and ${ }^{13} \mathrm{C}$ NMR spectral data, the molecular ion peak of 7 was established at $\mathrm{m} / \mathrm{z} 960$ consistent with the molecular formula of a salicyl tetraglycosidic ester $\left(\mathrm{C}_{47} \mathrm{H}_{76} \mathrm{O}_{20}\right)$. An ion peak generating at $m / z 137$ [O-1a fission, $\left.\mathrm{C}_{6} \mathrm{H}_{4}(\mathrm{O}) \mathrm{COOH}\right]^{+}$indicated the location of salicylic unit at one of the terminal of the molecule. The ion fragments produced at $\mathrm{m} / \mathrm{z} 295$ [O- $\mathrm{C}_{1}$ fission, $\left.\mathrm{C}_{20} \mathrm{H}_{39} \mathrm{O}\right]^{+}, 427$ [O$1 \mathrm{~d}$ fission, $\left.\mathrm{C}_{20} \mathrm{H}_{39} \mathrm{O}-\mathrm{C}_{5} \mathrm{H}_{8} \mathrm{O}_{4}\right]^{+}$, and 401 [O- $1 \mathrm{c}$ fission, $\left.\mathrm{C}_{6} \mathrm{H}_{5}(\mathrm{COOH})-\mathrm{C}_{5} \mathrm{H}_{8} \mathrm{O}_{5}-\mathrm{C}_{5} \mathrm{H}_{8} \mathrm{O}_{4}\right]^{+}$, suggested the diterpenic unit at another terminal and presence of four $\mathrm{C}_{5}$ sugar units in the molecule. The ${ }^{1} \mathrm{H}$ NMR spectrum of 7 displayed two one-proton double doublets at $\delta 7.66(J=2.8,8.5 \mathrm{~Hz})$ and $7.45(J=2.3,8.5 \mathrm{~Hz})$, and two one-proton multiplets at $\delta 7.35$ and 7.30 assigned to aromatic $\mathrm{H}-3$ adjacent to glycosidic oxygen, $\mathrm{H}-6, \mathrm{H}-5$, and $\mathrm{H}-4$ protons, respectively. Two one-proton doublets at $\delta 5.21(J=7.2$ $\mathrm{Hz})$ and $5.10(J=7.1 \mathrm{~Hz})$ and a two-proton doublets at $\delta 5.01$ $(\mathrm{J}=7.3 \mathrm{~Hz})$ assigned to sugar anomeric $\mathrm{H}-1 \mathrm{a}, \mathrm{H}-1 \mathrm{~b}, \mathrm{H}-1 \mathrm{c}$, and $\mathrm{H}-$

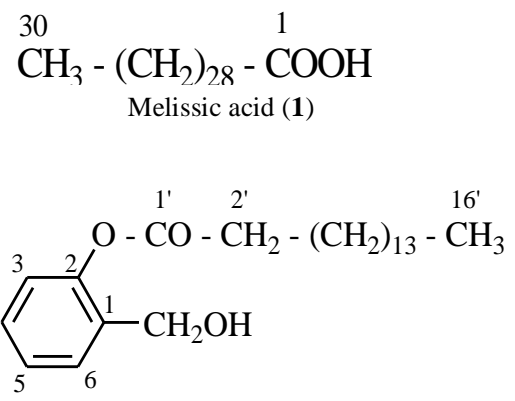

2-Palmityloxy benzyl alcohol (2)

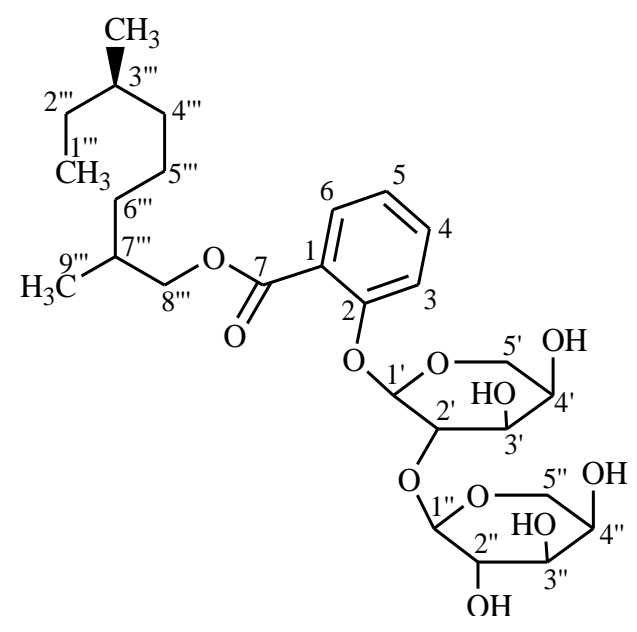

2-O-Diarabinosyl-8"'-geranilanyl salicylate (3) 1d protons, respectively. The other sugar protons resonated from $\delta$ 4.20 to 3.49. Four three-proton doublets at $\delta 0.94(J=6.8 \mathrm{~Hz}), 0.90$ $(J=6.0 \mathrm{~Hz}), 0.87(J=7.2 \mathrm{~Hz})$, and $0.84(J=6.8 \mathrm{~Hz})$, and a threeproton triplet at $\delta 0.79(\mathrm{~J}=6.8 \mathrm{~Hz})$ were accounted to secondary $\mathrm{C}$ 17', C-19', C-18' and C-20' and primary C-16' methyl protons, respectively. The remaining methine and methylene protons appeared between $\delta$ 2.28-1.16. The ${ }^{13} \mathrm{C}$ NMR spectrum of 7 displayed signals for aromatic carbons between $\delta 153.21$ to 127.67, carboxylic carbon at $\delta 180.36(\mathrm{C}-7)$, ester carbon at $\delta$ 171.63 (C-1'), anomeric carbons at $\delta 103.65$ (C-1a), 103.49 (C-1b), $101.74(\mathrm{C}-1 \mathrm{c})$ and $98.08(\mathrm{C}-1 \mathrm{~d})$ and the remaining sugar carbons between $\delta 83.15$ to 62.67 . Other methylene and methine carbons are resonated between $\delta 56.59-20.91$, primary and secondary methyl carbon appeared between $\delta$ 17.71-10.99. The presence ${ }^{1} \mathrm{H}$ NMR signals in the downfield region as multiplets at $\delta 4.03(\mathrm{H}-$ 2a), $\delta 3.97(\mathrm{H}-2 \mathrm{~b}), \delta 3.92(\mathrm{H}-2 \mathrm{c})$, and $\delta 4.20(\mathrm{H}-2 \mathrm{~d})$ and their respective carbon signals at $\delta 76.73(\mathrm{C}-2 \mathrm{a}), 75.22(\mathrm{C}-2 \mathrm{~b}), 74.77$ $(\mathrm{C}-2 \mathrm{c})$ and $85.15(\mathrm{C}-2 \mathrm{~d})$ suggested the $(2 \mathrm{a} \rightarrow 1 \mathrm{~b}),(2 \mathrm{~b} \rightarrow 1 \mathrm{c})$ and $(2 \mathrm{c} \rightarrow 1 \mathrm{~d})$ linkages between four sugar units and aliphatic chain at $\mathrm{C}-2 \mathrm{~d}$. On the basis of above discussion the compound $\mathbf{7}$ is structurally elucidated as 2-hydroxy benzoic acid-2- $O-\beta-D$ arabinopyranosyl-( $2 \mathrm{a} \rightarrow 1 \mathrm{~b})-O-\beta-D$-arabinopyranosyl-( $2 \mathrm{~b} \rightarrow 1 \mathrm{c})-O-$ $\beta$ - $D$-arabino- pyranosyl-(2c $\rightarrow 1 \mathrm{~d})-O-\beta$ - $D$-glucuranopyranosyl-2d$\left(2^{\prime}, 6^{\prime}, 10^{\prime}, 14^{\prime}\right.$-tetramethyl)- $n$-hexadecan-1'-oate. This is a new salicylic acid tetraglyosidic diterpenoid.

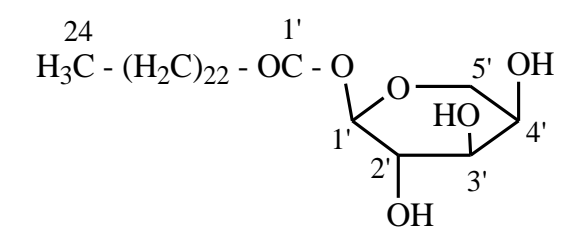

Lignoceryl $O-\beta$ - $D$-arabinoside (4)

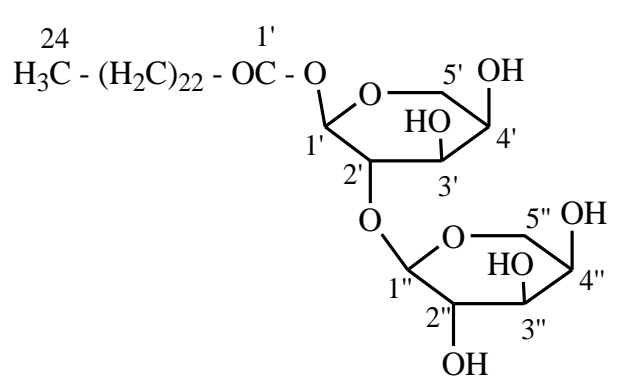

Lignoceryl diarabinoside (5)

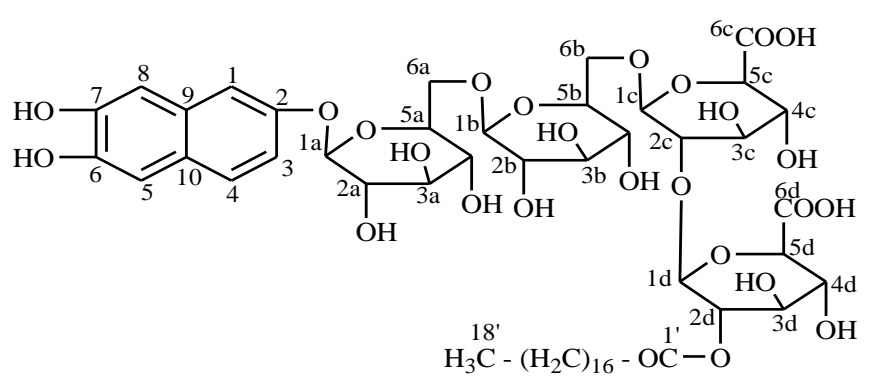

Trihydroxynaphthyl tetraglycosidic stearate (6) 


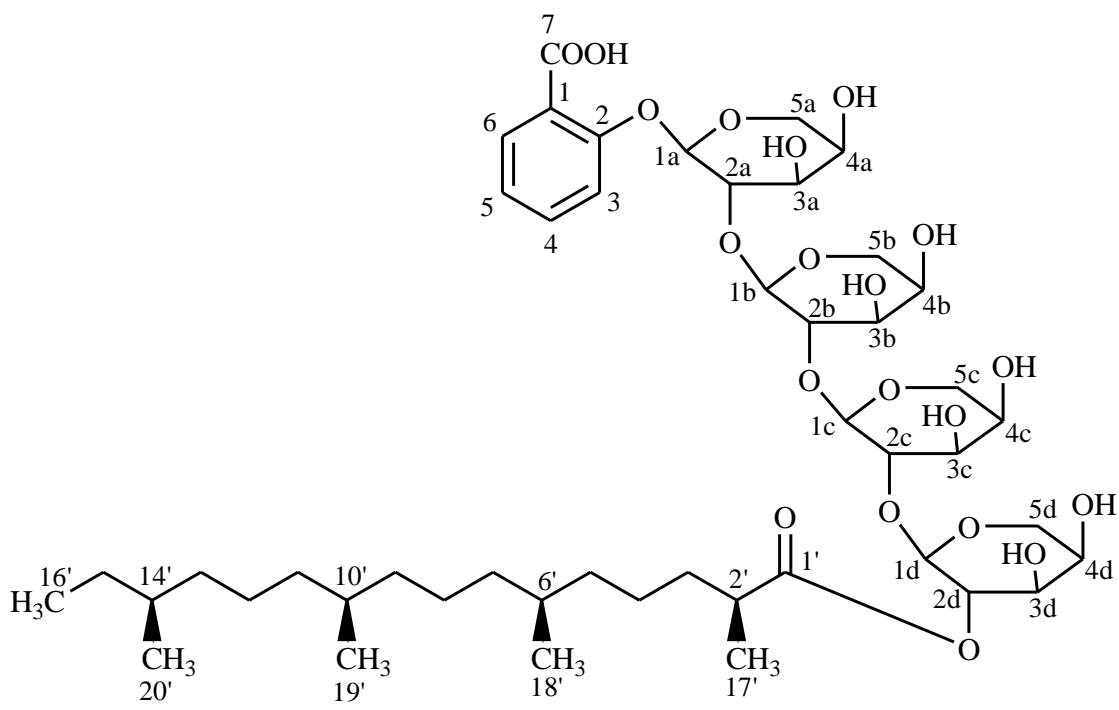

Salicylic acid-2-O- $\beta$-tetra-arabinosyl tetramethylhexadecanoate (7)

\section{CONCLUSION}

Plants have been used as medicine for humankind since ancient times in the indigenous systems and many tribal communities. They produced diverse range of novel bioactive secondary metabolites making them a prosperous therapeutic application. Isolation of six new crucially unmatched complex and potentially active secondary metabolites from this traditional medicinal herb has proven their ancient and folkloric utilization. Further chemical pattern of this plant could be used as reference compounds and establish a platform for authentication and quality control purposes in future.

\section{ACKNOWLEDGEMENT}

The authors are very thankful to Jamia Hamdard University, New Delhi, India, for providing research and instrumental facility such as recording to NMR and FT-IR spectral data.

\section{Financial support and sponsorship: NIL.}

Conflict of Interests: There are no conflicts of interest.

\section{REFERENCE}

Anonymous, 2005. Wealth of India, supplements, National Institute of Science Communication and Information Resources, Vol. 3, p. 152, CSIR, New Delhi 2003.

Anonymous, 2006. Wealth of India, supplements, National Institute of Science Communication and Information Resources, Vol. 1, p. 377-378, CSIR, New Delhi 2003.

Chibnall AC, Ernest FW, Alfred LL, tephen HP. The isolation of n-triacontanol from Lucerne wax. Biochemical Journal 1933; 27: 18851888.

Chopra RN, Nayar SL, Chopra SN, 2002. Glossary of Indian Medicinal Plants. New Delhi: National Institute of Science Communication and Information Resources (CSIR); pp. 122.
Duke JA, Bogenschutz-Godwin MJ, Du celliar J, Duke PK 2002. Hand Book of Medicinal Herbs, second ed. CRC Press, Boca Raton, pp. 314.

Fakim AG. Medicinal plants: Traditions of yesterday and drugs of tomorrow. Molecular Aspects of Medicine 2006; 27: 1-93.

Haq IU, Hussain M. Medicinal plants of Mansehra. Hamdard Medicus. 1993;36: 78-79.

Hussain SF, Shamma M. Parviflorine, a glycosidic spirobenzylisoquinoline alkaloid. Tetrahedron Letters 1980; 21: 19091912

Jameel M, Ali A, Ali M. Phytochemical investigation of the aerial parts of Fumaria parviflora Lam. Journal of Pharmaceutical and Biosciences 2014B ;2: 1-8.

Jameel M, Ali A, Ali M. Phytoconstituents from the aerial parts of Fumaria parviflora Lam. Journal of Advanced Pharmaceutical Technology \& Research 2014C ;5: 64-69.

Jameel M, Islamuddin M, Ali A, Afrin F, Ali M. Isolation, characterization and antimicrobial evaluation of a novel compound $\mathrm{N}$ octacosan $7 \beta$ ol, from Fumaria parviflora Lam. BMC complementary and alternative medicine 2014A ;14:98; $1-9$.

Khare CP, 2004. Indian Herbal Remdies: Rational Western Therapy, Ayurvedic and Other Traditional Uses, Botany. Germany: Springer-Verlag Berlin Heidelberg, pp. 225-226.

Kirtikar KR, Basu BD 1999. Indian Medicinal Plants, published by International Book Distributors; vol 1, 138-139.

Lidén M. Notes on Dionysia, Corydalis and Fumaria in Iran. Iran. J. Bot. 2000;8: 303-308.

Mabberley DJ, 2008. The Plant-book, Cambridge university press, Cambridge, $3^{\text {rd }}$ Edition, ISBN-13: 9780521820714.

Mossa JS, Al-Yahya MA, Al Meshal IA, 1987. Medicinal Plants of Saudi Arabia, $1^{\text {st }}$ ed. King Saud University Libraries Publications, Riyadh, ISBN: 9960-36- 476-3.

Nadkarni KM, 1967. Indian Materia Medica (third Ed). Popular Prakashan, Bombay pp. 560-561.

Naz I, Palomares-Rius JE, Saifullah, Blok V, Khan MR, Ali S, Ali S. In vitro and in planta nematicidal activity of Fumaria parviflora (Fumariaceae) against the southern root-knot nematode Meloidogyne incognita. Plant Pathology 2013; 62: 943-952.

Orhan I, Sener B, Musharraf SG. Antioxidant and hepatoprotective activity appraisal of four selected Fumaria species and their total phenol and flavonoid quantities. Exp Toxicol Pathol. 2010; 64: 205-209. 
Popova ME, Simanek V, Dolejs L, Smysl B, Preininger V. Alkaloids from Fumaria parviflora and Fumaria kralikii. Planta Med 1982;45: 120-122.

Rahman AU, Khati MK, Choudhary MI, Sener B. Chemical constituents of Fumaria indica. Fitoterapia 1992; 63:129-135.

Sousek J, Guedon D, Adam T, Bochorakova H, Taborska E, Valka I, Simanek V. Alkaloids and organic acids content of eight Fumaria Species. Phytochem. Anal. 1999;10: 6-11.

Suau R, Cabezudo B, Rico R, Najera F, Lopez-Romero JM. Direct determination of alkaloid contents in Fumaria species by GC-MS. Phytochemical Anal 2002;13: 363-367.

How to cite this article:

Jameel M, Ali A, Ali M., Identification of new compounds from Fumaria parviflora Lam. J App Pharm Sci, 2017; 7 (04): 053-060. 